\title{
It's All Around You: Range-Guided Cylindrical Network for 3D Object Detection
}

\author{
Meytal Rapoport-Lavie \\ Tel-Aviv University \\ laviemeytal@mail.tau.ac.il
}

\author{
Dan Raviv \\ Tel-Aviv University \\ daravatauex.tau.ac.il
}

\begin{abstract}
Modern perception systems in the field of autonomous driving rely on $3 D$ data analysis. LiDAR sensors are frequently used to acquire such data due to their increased resilience to different lighting conditions. Although rotating LiDAR scanners produce ring-shaped patterns in space, most networks analyze their data using an orthogonal voxel sampling strategy. This work presents a novel approach for analyzing $3 D$ data produced by 360-degree depth scanners, utilizing a more suitable coordinate system, which is aligned with the scanning pattern. Furthermore, we introduce a novel notion of range-guided convolutions, adapting the receptive field by distance from the ego vehicle and the object's scale. Our network demonstrates powerful results on the nuScenes challenge, comparable to current state-ofthe-art architectures. The backbone architecture introduced in this work can be easily integrated onto other pipelines as well.
\end{abstract}

\section{Introduction}

Robustness is a crucial requirement for visual perception solutions in autonomous driving systems. Such systems must be resilient to various lighting scenarios and withstand harsh weather conditions without compromising their performance. Therefore, extending the traditional RGB cameras with additional sensors, such as LiDAR, is a vital step towards achieving this goal.

The inclusion of depth information, which allows capturing the three-dimensional structure of the vehicle's environment, is a key feature for ensuring robustness while maintaining high accuracy. Modern LiDAR acquisition sensors provide meaningful information not just for avoiding imminent collisions but to perceive the environment as good as image-based data and even surpass it under poor lighting conditions.

In the field of autonomous driving, there is a variety of methods to handle point clouds. The most common and

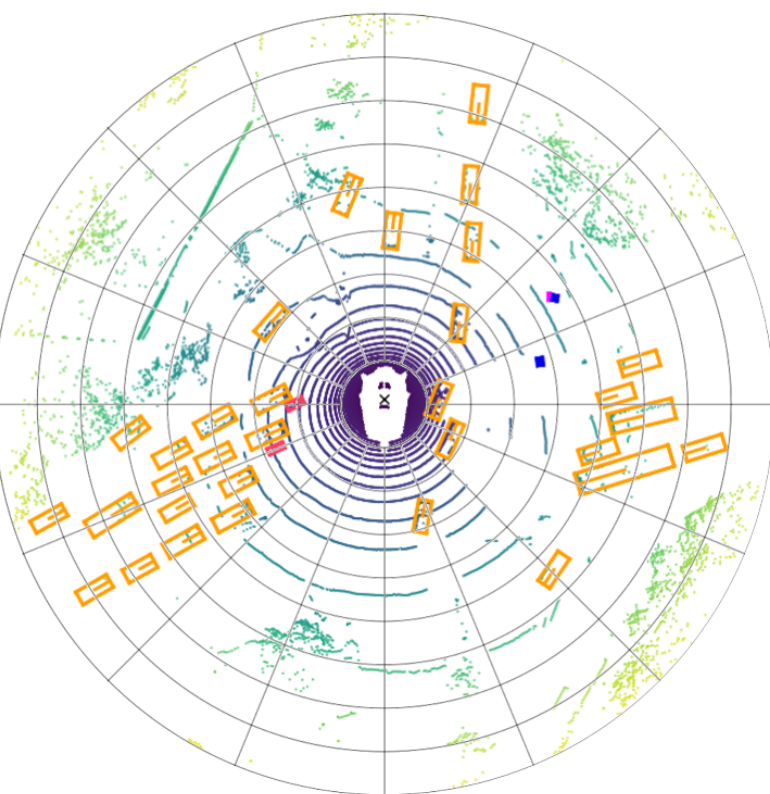

Figure 1. Top-View of a nuScenes dataset sample with Ground Truth detections. First, it can be easily observed that the LiDAR sensor has a ring-shaped pattern output. Second, the Cylindrical Coordinate system maintains 9 neighbors and 27 neighbors per voxel (including itself), in 2D and 3D respectively, the same as the classical Cartesian system. This enables us to employ the classical convolution layers without almost any alteration.

efficient approach is to transform the point-cloud into a regular representation, such as 2D bird-eye-view (BEV) voxels $[13,7,27,14]$ or 3 D voxels $[24,31,8,26]$. Following the architecture of PointNet and PointNet++ [4, 21], some works have proposed replacing the voxel-based method with a point-based one. This approach, however, was found to be computationally intensive. Recent papers have suggested combining both point-based and voxel-based methods into a single network $[23,16]$. However, whether combining point-based methods or not, in 2D or 3D, these methods use the Cartesian coordinate system as their grid. 
In this paper, we argue that projecting the points onto a Cartesian space disregards the circular nature of the raw data provided by a rotating emitter. We show that in order to perceive and preserve the additional information gained from a LiDAR sensor, one may leverage a more appropriate system, namely the Cylindrical coordinates system. Furthermore, the Cylindrical coordinates system allows us to align the entire network towards the sensor's point of view. Intuitively, the closer an object is to the ego vehicle, the more points correspond to it in the point cloud. Therefore we suggest adjusting the size of the voxels to the distance from the sensor, as is done naturally by the Cylindrical space. To do so, we propose a novel guiding unit, which orchestrates convolutions by their range from the ego vehicle. We further explore the challenges for a "self-oriented" network to learn angle and velocity information and present the necessary solutions to overcome them successfully.

Our main contributions are as follows:

- We present the first end-to-end cylindrical coordinates perception architecture for autonomous systems, including orientation and velocity estimation.

- We present a novel range-guided convolution block to adjust the network's receptive field according to an object's range and scale, and adapting its learned features by its distance from the ego-vehicle.

- We provide new insights, challenges and solutions for training deep networks in cylindrical coordinates.

- Our network achieves comparable results to current state-of-the-art networks on the competitive nuScenes 3D object detection benchmark.

\section{Related works}

Cartesian Based 3D Object Detection Methods: Most state-of-the-art methods for 3D object detection project the point clouds onto a Cartesian coordinate grid. Earlier approaches employed the BEV projection to utilize efficient $2 \mathrm{D}$ convolution layers to process the point cloud data $[13,7]$. In a later work, VoxelNet [31] was first to introduce 3D voxels in the field of object detection, whereas SECOND [26] improved this method by applying sparse 3D convolutions to accelerate running time. Most cutting edge methods [24, 23], including the previous and current nuScenes' state-of-the-art, CBGS and CenterPoint [32, 28], have adapted the practice of processing the point cloud through sparse 3D convolutions after projecting it to the traditional Cartesian voxel space.

Cylindrical / Spherical Based Methods: Only recently, PolarNet [30] has suggested quantizing LiDAR data in

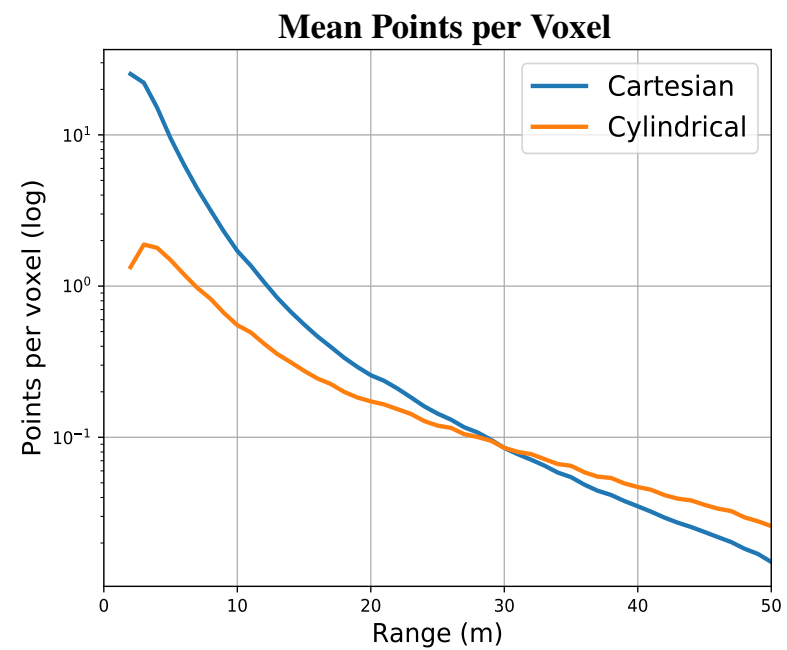

Figure 2. Mean number of points per voxel (logarithmic) as a function of range. There is a high inverse correlation between the number of points and their range from the sensor. Since the Cartesian voxels are of the same size in all ranges, we lose precision in the nearby range. On the other hand, in Cylindrical coordinate we receive a better spatial spread.

a non Cartesian coordinate system. Their Polar-BEV network, designed for semantic segmentation on the SemanticKITTI [1] challenge, have shown improvement with respect to traditional Cartesian methods. Unlike semantic segmentation tasks, 3D detection challenges, such as nuScenes, require the processing of additional object orientation and velocity features. As direction information plays a significant role in such tasks, they are directly affected by the change in the coordinate system and therefore need to be treated with special care.

3D Object Detection Methods with Focus on Range: LaserNet [19] has introduced the concept of range-image input to the field of 3D detection, naturally emphasizing an object's distance from the ego vehicle as one of its key features. Despite its computational efficiency, it was generally outperformed by voxel-based methods. A later publication [2] suggested a method to address range-image's problem of scale variance, the fact that near distance objects appear larger in the image view. They applied dilated convolution $[5,25]$ directly to the range-image input, adjusting each pixel's dilation rate as a function of its range.

In two recent papers CVCNet and RangeRCNN [22,16] tried to benefit from both range view and BEV. CVCNet [22] proposed a pair of cross-view transformers to transform the feature maps into the other view and offered a new voxel representation, Hybrid-Cylindrical-Spherical (HCS) voxels, which enables them to extract features for both Range view and BEV in a unified coordination system. RangeRCNN [16] argued that although range-image 

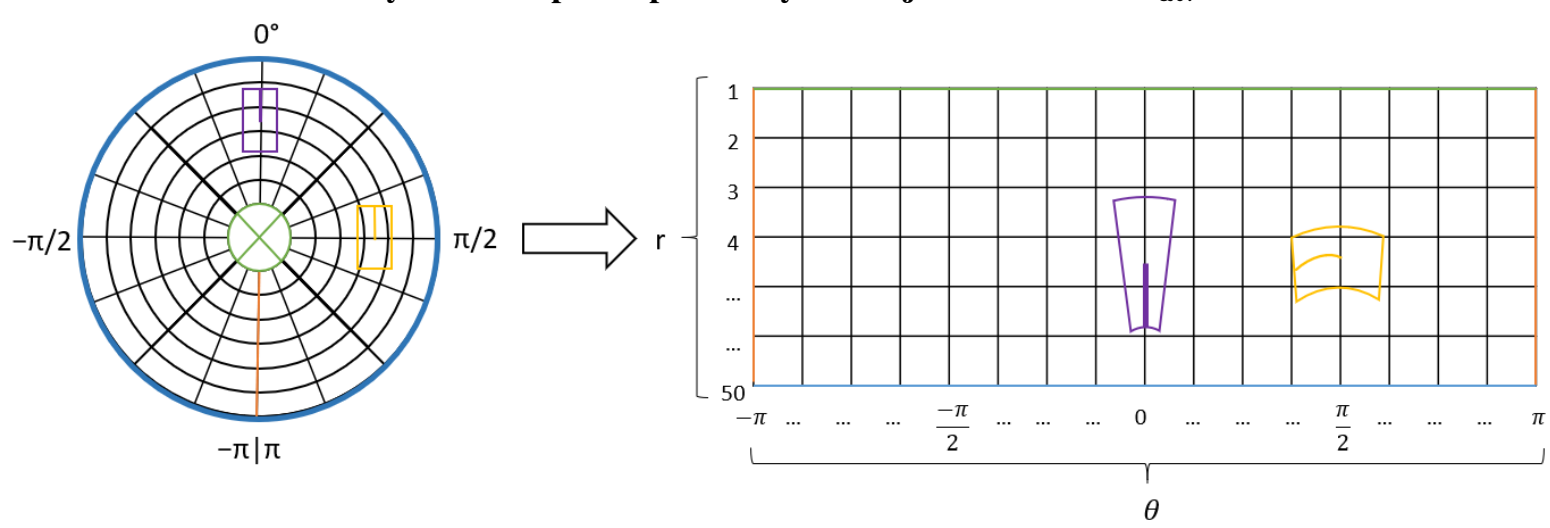

Figure 3. Illustration of the Cylindrical map translated into the network's 2D array. Here shown two identical cars placed at the same distance from the ego vehicle but have different $\theta_{\text {center. }}$. Since we are working from the sensor's point of view, we can see that it translates differently to the $2 \mathrm{D}$ array. The network observes the yellow car from the side as the purple car from behind, just as the sensor/driver does.

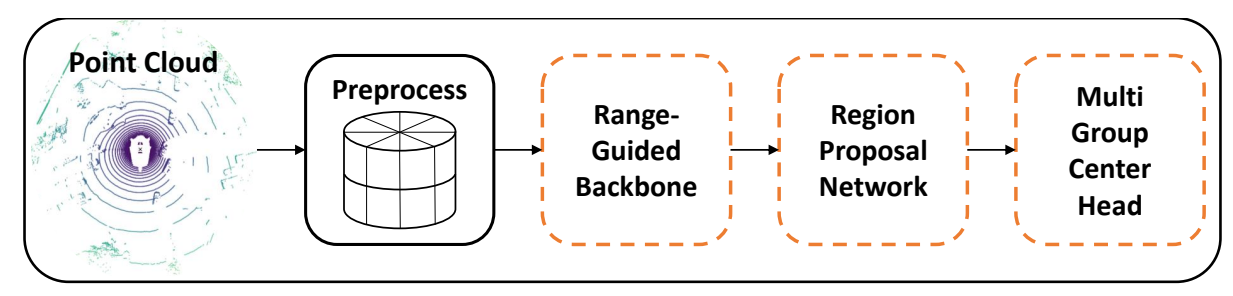

Figure 4. A diagram illustrating the main components of the network architecture. Orange dashed lines indicating deep networks.

is insufficient by itself, incorporating it as a source of initial feature extraction, before transforming the data into BEV architectures can be effective. This method of enhancing BEV with range-image features achieves state-of-the-art results on the KITTI dataset [10].

In our work, we use range information to guide the 3D convolution layers. In this manner, we derive additional benefit from its data as an initial feature, allowing the network to comprehend objects differently depending on their distance from the ego vehicle. Furthermore, we use range information to manage the receptive field of the voxels, for overcoming their scale variance in the Cylindrical coordinate system.

\section{Challenges in Cylindrical Coordinates}

The Cylindrical-coordinate grid differs from the Cartesian in many aspects. First and foremost it is a self-oriented system. Consequently, identical objects placed at different positions relative to the sensor location will be presented differently along the network's channels. For this reason, in order to adapt the network to the sensor's point of view, certain challenges have to be addressed.

In contrast to Cartesian coordinates BEV, the size of a Cylindrical voxel varies depending on its range. Thus two identical objects can spread over a different number of voxels, depending on their center location relative to the ego vehicle. Likewise, the orientation of an object toward the ego vehicle might also affect the number of voxels it occupies in the Cylindrical grid. In general, objects spread on a larger amount of voxels, require a wider receptive field in order to detect them.

The Cartesian and Cylindrical-coordinate systems differ in the way they perceive the orientation of objects. The network's point of view can be equated with the perspective of a passenger in the ego-vehicle, observing two identical cars, one of which is in front of the vehicle while the other is on its side. As illustrated in Figure 3, both yellow and purple cars, are of the same size, facing the same way, and at the same range. However, the difference in their $\theta$-centers causes them to be mapped differently to the network's input arrays with an emphasis on the direction. As shown, the purple car and the yellow ones are no longer facing the same direction in the 2D input array of our network. In order for the network to learn its orientation accordingly, it should be modified in a proper manner.

The same principle applies to the velocity's direction. For example, an object moving along a certain axis will seem to the Cartesian-based network to be moving along that axis regardless of its location on the map. This behav- 


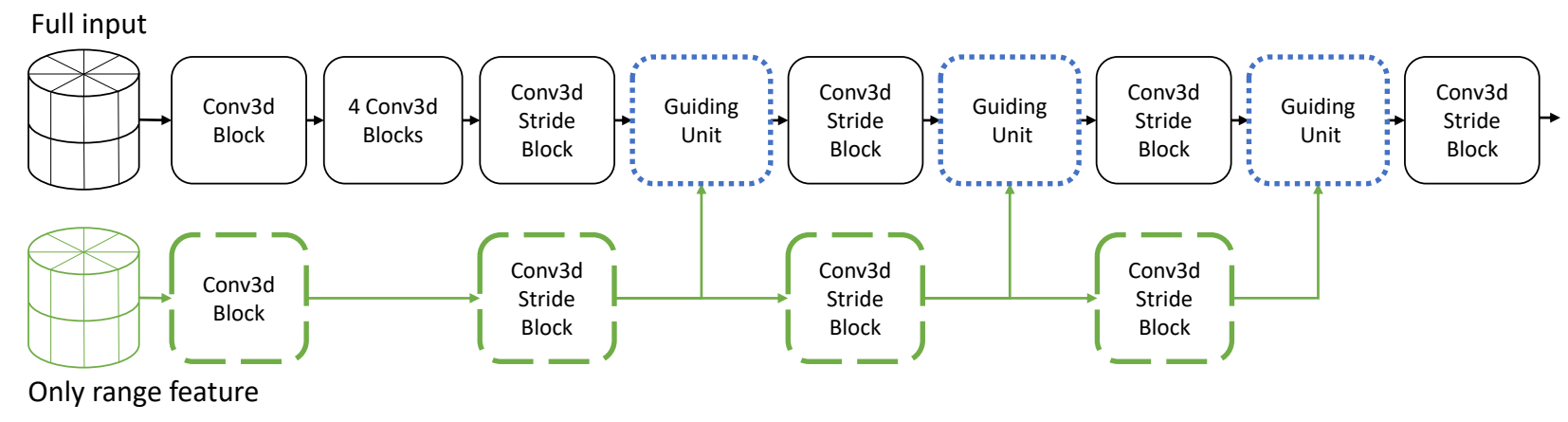

Figure 5. Illustration of the Range-Guided Backbone: The guided backbone is shown in black, the range guiding backbone is shown in a green dashed line and the guiding unit are shown in blue doted lines. We added a parallel range feature branch in order to guide a simple backbone consisting of convolution layers.

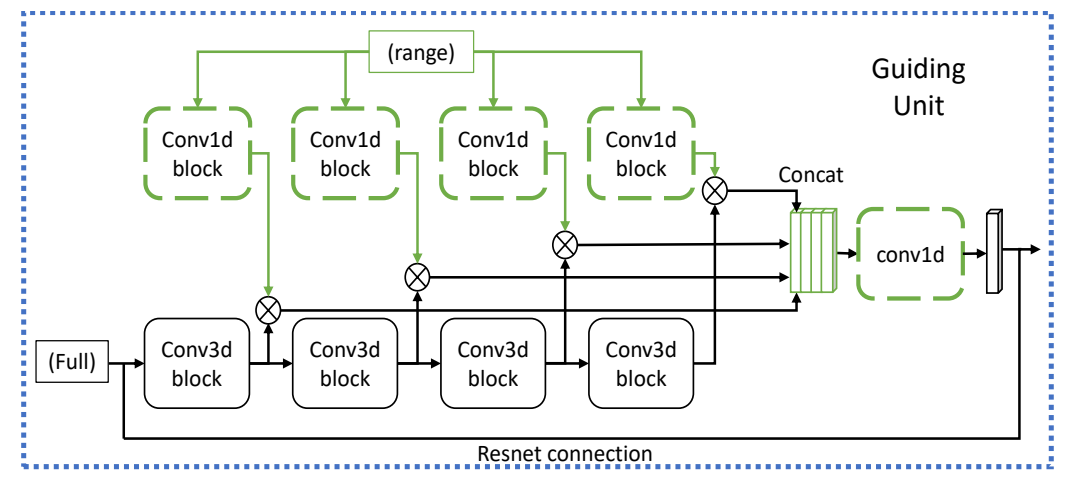

Figure 6. Illustration of the Guiding Unit: On the range input we use four separate 1D convolutions. The output of each one is multiplied by the output of its parallel convolution layer on the full input and then concatenated together. In order to achieve the original number of features, we apply 1D convolution with a residual [12] connection.

ior manifests because the axes of Cartesian coordinates are aligned to those of every other object on the map. However, for the Cylindrical coordinates, this is not the case, and therefore additional adjustments are necessary.

Additionally, since the $\theta$ axis is inherently cyclical ( $-\pi$ is also $\pi$ ), objects may spread simultaneously over voxels at both the beginning and the end of the network's input arrays. This is a further deviation from the Cartesian system that needs to be taken into account in order to accomplish the transition between the two systems.

In conclusion, transferring from a standard coordinate system towards a self-oriented one reveals our networks' unique challenges. To take full advantage of the many benefits offered by Cylindrical coordinates, fundamental changes are needed throughout the network's architecture and the outputs that are being learned.

\section{Method}

Our network consists of three learning blocks: (1) Range-Guided Backbone, which receives the 3D voxelized input and constructs an output of a flattened 2D map by utilizing our novel guiding units, (2) Region Proposal Network and (3) a Multi-Group Center Head that outputs a heat-map per class and a variety of predicted parameters for each detected object. Adapting the entire solution to handle the cyclical nature of the $\theta$ axis called for modifications across all three.

Input: Although the raw output of a LiDAR sensor is given in range and two angles, the nuScenes dataset provides the point cloud sweeps already transformed to Cartesian coordinates. Each point is assigned with two additional features: intensity, which is taken directly from the sensor, and $\Delta t$, which relates to the lag-time between each sweep to its key-frame sweep. We followed the rules of the nuScenes detection benchmark by taking ten sequential samples (totaling 0.5 seconds). We then quantize it according to the cylindrical voxel resolution and average on each voxel across all features. 

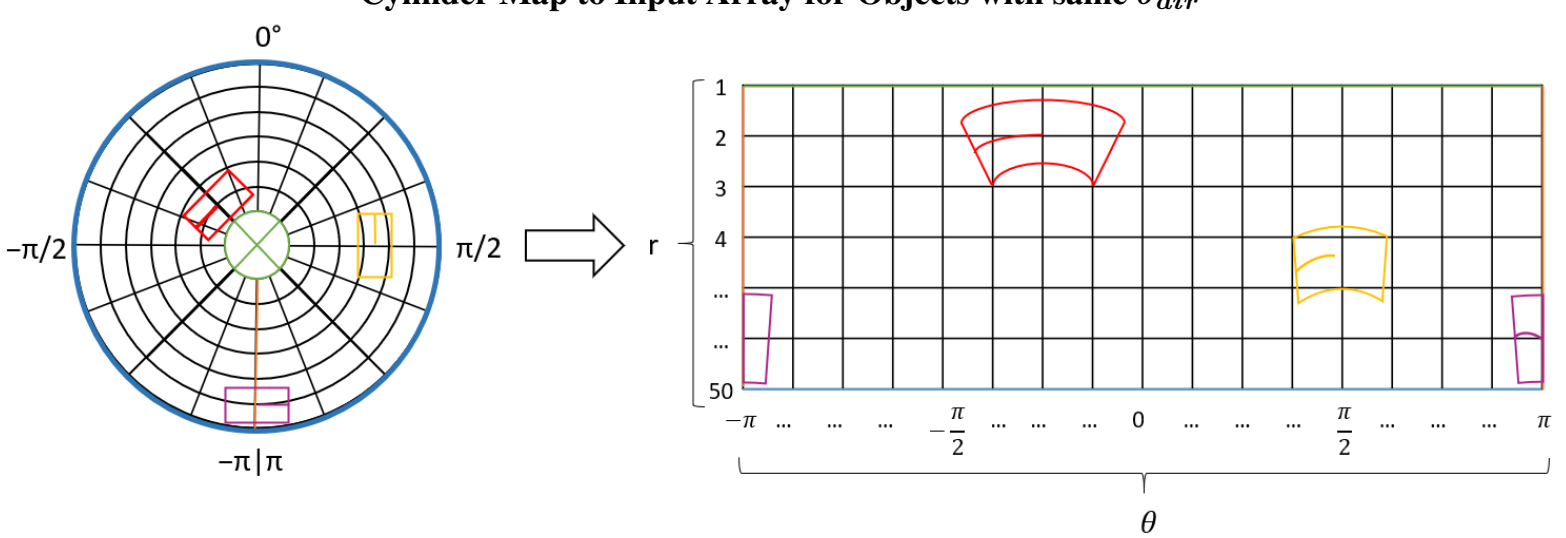

Figure 7. Illustration of the Cylindrical map translated into the network's 2D array. Here shown three identical cars of the same size, but with different centers. They also have different $\theta_{d i r}$ but the same $\bar{\theta}_{d i r}$ (defined in equation (3)), as viewed from the sensor's perspective. We can also observe here that the closer the vehicle is to the sensor, the more voxels in the $\theta$ axis it occupies. We also see here the circularity character of the $\theta$-axis where the magenta car is spread on both sides of the $2 \mathrm{D}$ array.

Range-Guided Backbone: In the first block of our proposed solution, we introduce the range-guided backbone. Since similar objects appear across different bins depending on the range, we propose to control the convolutions along the main pipeline using a range guided mechanism.

The motivation for range guidance is two-fold; First, the LiDAR transmitter releases rapid pulses of laser light across a pre-defined spatial pattern. Using a synced sensor, it measures the time it takes for each pulse to reflect back from the object it hits. Then, we receive multiple hits per object, which depends on its distance. Meaning, nearby objects will be over-represented in contrast to far away objects, as can be seen in Figure 2 leading to significant deviation in close and distant objects statistics. The second reason relates to the voxel size. As noted earlier (see Section 3), the voxel size in BEV increases by the range in Cylindrical coordinates. Which means nearby objects need a wider receptive field than far away objects.

The main guided backbone was inspired by CBGS [32] who followed the work of SECOND[26]. The range guiding backbone is made of a convolution pipeline sourced by range only matrix (meaning only the range feature of each voxel), controlling the number and weights of consecutive convolutions running on the main pipeline. See Figure 5 for visual aid.

Specifically, the range backbone is parallel to the main pipeline. In each guiding unit, the output of each 3D convolution running on the main pipeline is multiplied by the output of a $1 \times 1$ convolution which directly operates on the range matrix. The product of four such multiplications are concatenated and then reduced in dimensions by a $1 \mathrm{x}$ 1 convolution operator. The design of the guiding unit allows the neural network to learn different receptive fields, while the 3D convolution layers in between contribute to its learning capabilities of the spatial information of neighboring voxels. In our Cylindrical network, this backbone will serve mainly the far range where the receptive field needs to be smaller.

RPN: We have found that the RPN adopted by CBGS after following the VoxelNet [32,31] method is suitable for our network as well.

The RPN consists of two levels of features. On both levels, we use multiple 2D convolutions, but on one of them, we first use a single convolution with stride 2 , and in the end, we apply deconvolution to return it to the original map size. Both levels then get concatenated together to obtain a higher feature map.

Multi Group Center Head: For this block, we follow the head architecture of CenterPoint [28]. In order to make use of the block, some adjustments were needed to overcome the aforementioned challenges using Cylindrical coordinates.

The original head outputs a K-channel 2D heatmap to indicate the centers of the K-class objects in the $\mathrm{x}$-y plane. In addition to the heatmap, the network outputs ten different scalars for each detection: $(d x, d y)$ which is the delta to the object's center from the heatmap coordinates, $(w, l, h)$ which indicate the object width, length and height, $\left(z_{\text {center }}\right)$ for the object's center in the Z-axis, $\left(v_{x}, v_{y}\right)$ for the object's velocity in $\mathrm{X}$ and $\mathrm{Y}$ axes respectively and $\left(\cos \theta_{d i r}, \sin \theta_{d i r}\right)$ where $\theta_{d i r}$ is the heading angle of the object.

The ground truth heatmaps for training are built by rendering a Gaussian around each object's center. CenterPoint 
[28] uses symmetric 2D Gaussian since the $\mathrm{X}$ and $\mathrm{Y}$ axes share the same resolution, and calculate the radius using the following equation:

$$
\sigma=\max (f(w l, r), \tau)
$$

Where $f$ is the radius function defined in CornerNet [15], $\tau$ is the smallest allowable Gaussian radius and $w, l$ are the width and length of the detected object in number of voxels, meaning the result of $w$ and $l$ divided by the voxel's size.

In our setup, we can not use symmetric Gaussian anymore thus we split it into 2D Gaussian with different radii for each axis which are calculated separately by equation (1). We then calculate the size of a voxel in the theta-axis by the following formula:

$$
V_{\theta \text { size }}=2 r_{\text {center }} \sin \left(\theta_{\text {center }} / 2\right)
$$

Where $r_{\text {center }}$, and $\theta_{\text {center }}$ are the coordinates of the object's center in the $\mathrm{r}$ and $\theta$ dimensions respectively.

While the transformation of the heatmap coordinates and the deltas from Cartesian to Cylindrical coordinates is quite simple, the heading angle and velocity outputs are much more complex and require a deep understanding of the network's view.

The $\theta_{d i r}$ in which our Cylinder-network observes the object depends not only on the $\theta_{\text {dir }}$ of the object but also on the $\theta_{\text {center }}$ of the object. An object facing forward with $\theta_{\text {center }}=\pi / 2$ will be interpreted on the $2 \mathrm{~d}$ map the same way an object-centered right in front of the ego-vehicle and facing $\theta_{d i r}=-\pi / 2$ will see Figure 7 . Therefore, the $\theta_{d i r}$ we wish to learn is as follows:

$$
\bar{\theta}_{d i r}=\theta_{\text {dir }}-\theta_{\text {center }}
$$

Where $\theta_{\text {center }}$ is the $\theta$ coordinate of the object's center.

From the calculation of $\bar{\theta}_{d i r}$, we gain significant added value over the Cartesian coordinate system. As demonstrated in Figure 8, points are distributed differently between similar cars with respect to their $\theta_{\text {center. }}$. Although the two cars share identical $\theta_{d i r}$ in the classic system, the points are scattered on different parts of each vehicle. As the blue bound car is mostly "hit" on its front, the points "hitting" the green marked car mainly concentrate on its left side. In our modified system, for instance, when most of the object's cloud points cover its rear, the value of its $\bar{\theta}_{d i r}=0$, when the majority of the points appear on its right side, $\bar{\theta}_{d i r}=\pi / 2$, and so forth. We believe it allows our system a more adequate comprehension of the scene.

Now we can separate the new $\bar{\theta}_{d i r}$ into two orthogonal components:

$$
\begin{aligned}
& \theta_{\theta}=\sin \left(\bar{\theta}_{d i r}\right) \\
& \theta_{r}=\cos \left(\bar{\theta}_{d i r}\right)
\end{aligned}
$$

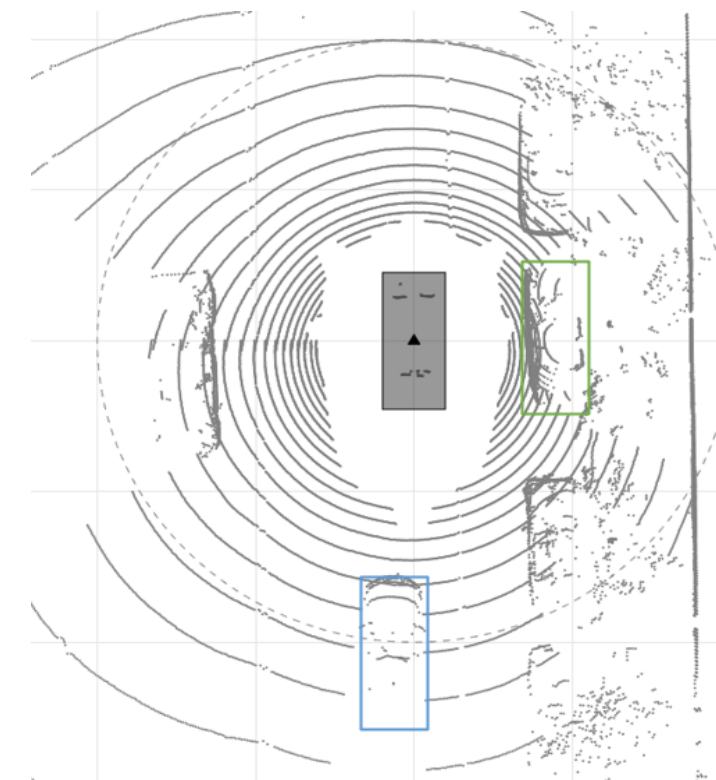

Figure 8. A single frame of nuScenes scene, demonstrating the Cartesian system's drawback: Although the green and blue cars share many characteristics, in particular that they both have a similar orientation - the pattern in which they are covered with point clouds is significantly different. By applying the modifications described in equation 3 , this difference no longer poses a challenge to our Cylindrical network.

For each detection, one is also required to output the object's velocity. As opposed to $\theta$, the required velocity for this benchmark is already divided into the classical coordinates, meaning the metrics of this benchmark evaluates $v_{x}$ and $v_{y}$ and reported as ground truth. Therefore an extra step is required:

$$
\begin{aligned}
& V_{a b s}=\sqrt{v_{x}^{2}+v_{y}^{2}} \\
& V_{d i r}=\arctan \left(\frac{v_{x}}{v_{y}}\right)
\end{aligned}
$$

Where we use $V_{x}$ as the numerator and $V_{y}$ as the denominator to be aligned with nuScenes coordinate system. We then apply the same transformation we did in equation (3) for velocity:

$$
\bar{\theta}_{\text {velocity }}=V_{\text {dir }}-\theta_{\text {center }}
$$

We then to divide that into two learnable outputs according to our self-cylindrical coordinates as before:

$$
\begin{aligned}
& V_{\theta}=V_{\text {abs }} \sin \left(\bar{\theta}_{\text {velocity }}\right) \\
& V_{r}=V_{\text {abs }} \cos \left(\bar{\theta}_{\text {velocity }}\right) .
\end{aligned}
$$




\begin{tabular}{|lcccccc||c||}
\hline Method & $m A P$ & $m A T E$ & $m A S E$ & $m A O E$ & $m A V E$ & $m A A E$ & $N D S$ \\
\hline CBGS [32] & 0.499 & 0.335 & 0.256 & 0.323 & $\mathbf{0 . 2 5 1}$ & 0.197 & 0.613 \\
CenterPoint [28] & $\mathbf{0 . 5 9 1}$ & $\mathbf{0 . 2 7 7}$ & $\mathbf{0 . 2 5 1}$ & $\mathbf{0 . 2 6 9}$ & $\underline{0.258}$ & $\underline{0.189}$ & $\mathbf{0 . 6 7 1}$ \\
Ours & $\underline{0.576}$ & $\underline{0.283}$ & $\underline{0.253}$ & $\underline{0.291}$ & $\underline{0.268}$ & $\underline{\mathbf{0 . 1 8 0}}$ & $\underline{0.661}$ \\
\hline
\end{tabular}

Table 1. nuScenes Experiment Results on Validation set: The results of CBGS and CenterPoint as shown on their github. Top results are in bold, second place is underlined.

\begin{tabular}{|lcccccc||c||}
\hline Method & $m A P$ & $m A T E$ & $m A S E$ & $m A O E$ & $m A V E$ & $m A A E$ & NDS \\
\hline CBGS [32] & 0.528 & 0.300 & 0.247 & $\underline{0.379}$ & $\underline{0.245}$ & 0.140 & 0.633 \\
CVCNet single [22] & 0.558 & 0.300 & 0.248 & 0.431 & 0.269 & $\mathbf{0 . 1 1 9}$ & 0.642 \\
CVCNet single V2 [22] & 0.553 & 0.300 & 0.244 & 0.389 & 0.268 & $\underline{0.122}$ & 0.644 \\
MMDetection3D [29] & 0.575 & 0.316 & 0.256 & 0.409 & $\mathbf{0 . 2 3 6}$ & 0.124 & 0.653 \\
HotSpotNet-0.1m [6] & $\underline{0.593}$ & 0.274 & $\mathbf{0 . 2 3 9}$ & 0.384 & 0.333 & 0.133 & 0.660 \\
CenterPoint single[28] & $\mathbf{0 . 6 0 3}$ & $\mathbf{0 . 2 6 2}$ & $\mathbf{0 . 2 3 9}$ & $\mathbf{0 . 3 6 1}$ & 0.288 & 0.136 & $\mathbf{0 . 6 7 3}$ \\
\hline Ours & 0.585 & $\underline{0.272}$ & $\underline{0.243}$ & 0.383 & 0.293 & 0.126 & $\underline{0.661}$ \\
\hline
\end{tabular}

Table 2. nuScenes Experiment Results on Test set: Top results for single model on the LiDAR track, taken from the nuScenes website. Top results are in bold, second place is underlined

Losses: Following CenterPoint[28] we use $L_{1}$ regression for the local offset $(d r, d \theta)$, orientation $\left(\theta_{r}, \theta_{\theta}\right)$ as declared on equation (4) and velocity $\left(V_{r}, V_{\theta}\right)$ as declared on equation (7). Additionally we use log-space $L_{1}$ regression for size $(w, l, h)$ and $z_{\text {center }}$. To supervise the heatmap we use a focal loss[17] with $\alpha=2$ and $\beta=4$.

The overall loss of our network is as follows:

$$
L=L_{\text {heatmap }}+\lambda_{\text {reg }} L_{\text {reg }}
$$

Where $L_{\text {heatmap }}$ is the focal loss for the heatmap and $L_{r e g}$ and $\lambda_{\text {reg }}$ are the regression losses mentioned above and their loss weight accordingly.

Circular Padding: We address the circularity of the $\theta$ axis of the neural network by substituting zero-padding with circular padding for the $\theta$-axis in all of the convolution layers throughout the entire network, both 2D and 3D.

By applying all of the above methodologies, we have completely converted the network to operate on selfcylindrical coordinates.

\section{Experiments}

Dataset: We evaluate our method on the nuScenes dataset for 3D object detection [3]. The dataset consists of 700 training scenes, 150 validation scenes and a test set of 150 scenes, each 20s long with LiDAR frequency 20 FPS. The latest nuScenes benchmark requires the detection of 10 different classes: car, bus, construction vehicle, trailer, truck, pedestrian, motorcycle, bicycle, traffic cone and barrier.
Evaluation Metrics: The nuScenes Detection Score (NDS) is a weighted sum of mean Average Precision (mAP) [9] and several True Positive (TP) metrics. The $\mathrm{mAP}$ is calculated as an average of four $\mathrm{mAP}$ with distance threshold of: $(0.5 \mathrm{~m}, 1 \mathrm{~m}, 2 \mathrm{~m}$ and $4 \mathrm{~m})$, and the TP metrics are calculated between the prediction and its matched ground truth the under the $2 m$ threshold:

1. Average Translation Error (ATE): The Euclidean distance in meters between the two centers.

2. Average Scale Error (ASE): Calculated as (1 - IOU) after aligning the centers and orientation of both.

3. Average Orientation Error (AOE): Smallest yaw angle difference $\left(\theta_{\text {dir }}\right)$.

4. Average Velocity Error (AVE): Absolute velocity error for $v_{x}$ and $v_{y}$ separately, measured in meters per second.

5. Average Attribute Error (AAE): Calculated as 1 - acc, where acc is the attribute classification accuracy.

The final NDS is calculated as follows:

$$
N D S=5 m A P+\sum_{i=1}^{5} \max \left(1-T P_{i}, 0.0\right)
$$

\subsection{Implementation details}

Preprocess: Our implementation is based on the opensourced code of CenterPoint ${ }^{1}$ implemented using the PyTorch [20] framework. Following the nuScenes Benchmark, we set the detection range to $[1 \mathrm{~m}, 53.8 \mathrm{~m}]$ for the 


\begin{tabular}{|ccccccccccc||c||}
\hline CC.mod. & $0.5 m$ & $1 m$ & $2 m$ & $4 m$ & $m A P$ & $m A T E$ & $m A S E$ & $m A O E$ & $m A V E$ & $m A A E$ & $N D S$ \\
\hline $\mathrm{X}$ & 0.468 & 0.561 & 0.618 & 0.648 & 0.574 & $\mathbf{0 . 2 8 2}$ & $\mathbf{0 . 2 5 0}$ & 0.314 & 0.312 & 0.185 & 0.652 \\
$\mathrm{~V}$ & $\mathbf{0 . 4 6 9}$ & $\mathbf{0 . 5 6 2}$ & $\mathbf{0 . 6 2 3}$ & $\mathbf{0 . 6 5 1}$ & $\mathbf{0 . 5 7 6}$ & 0.283 & 0.253 & $\mathbf{0 . 2 9 1}$ & $\mathbf{0 . 2 6 8}$ & $\mathbf{0 . 1 8 0}$ & $\mathbf{0 . 6 6 1}$ \\
\hline
\end{tabular}

Table 3. Ablation Experiment on nuScenes Validation set: Cylindrical-coordinate modifications (CC.mod.) refers to modification explained on subsection Multi Group Center Head under section Method 4 for both orientation $\left(\bar{\theta}_{\text {dir }}\right)$ and velocity $\left(\bar{\theta}_{\text {velocity }}\right)$. Notice, how our CC.mod. significantly improves performance for both, orientation (mAOE) and velocity (mAVE).

range axis, $[-\pi, \pi]$ for the $\theta$ axis and $[-3 m, 5 m]$ for the $\mathrm{Z}$ axis. Utilizing the structure of the cylindrical coordinates, we do not need extra spaces in our input map, in order to detect an object within the required $50 \mathrm{~m}$ range.

With the choice of $[0.075 \mathrm{~m}, \pi / 600 \mathrm{rads}, 0.2 \mathrm{~m}]$ for the voxels size in each axis respectively, our network input size is $704 \times 1200 \times 40$ voxels. Max point per voxel was set to 10 and the max number of voxels was set to $150 \mathrm{k}$. In our range-guided backbone we use four times stride 2 for the range axis, and three times for the $\theta$ axis, resulting in 88 x 300 output size. Due to the cylindrical shape of our output map, it is almost 20\% smaller than CenterPoint's [28] output map.

We adopt the class-balanced sampling and class-grouped heads of CBGS [32] to address the class imbalance of nuScenes dataset. We also conduct CBGS' augmentations on the original point-cloud in XYZ coordinates: global rotation in the range of $[-\pi / 8, \pi / 8]$, global scaling with random scale factor between $[0.95 \mathrm{~m}, 0.95 \mathrm{~m}]$, translation within the range of $0.2 \mathrm{~m}$ for each axis and random flip for $\mathrm{x}$ and $\mathrm{y}$-axis.

Training: We train the model with a batch size of 56 for 20 epochs on 8 RTX8000 GPUs. We have chosen adamW [18] optimizer with one-cycle policy [11], LR max 0.0035 with division factor 10 , momentum from 0.85 to 0.95 , weight decay 0.01 and chose $\lambda_{\text {reg }}$ to be 0.25 . All zeropadding in $\theta$ dimension was replaced with circular-padding and the size of the kernel was increased to 5 (instead of 3 ) along the backbone, to obtain a larger maximum reception field in this dimension.

Testing: During inference, we keep the top 500 detection proposals per sample. Consequentially, we filter out all proposals with a detection score lower than 0.1 and execute Non-maximum Suppression (NMS) with an IOU threshold of 0.1 and a max output of 83 proposals per group. We use CenterPoint [28] flip-test method but instead of using three additional flips (y-flip, $x$-flip, and $x y$-flip) to the original input, we use only one (y-flip) resulting in a reduced inference time by a factor of two.

\footnotetext{
1https://github.com/tianweiy/CenterPoint
}

\subsection{Results}

We present our results on both nuScenes validation and test sets. The test results were obtained from nuScenes official evaluation server, where test sample annotations remain hidden. Our single model on the LiDAR track outperforms all methods but a single one. Our network achieved a higher score than both of CVCNet [22] submissions, which is the only other method that did not use Cartesian coordinates.

\subsection{Ablation studies}

We further conduct our ablation studies on the nuScenes validation set.

First, we demonstrate the significance of our Cylindricalcoordinate modifications detailed in Multi-Group Center Head on section 4. Table 3 shows the result for two identical networks, trained in the same way, both utilizing circular padding. The first network has employed our velocity and orientation modifications, while the other network did not. As expected, the most significant improvement in the first network's performance can be seen in the velocity and orientation metrics. Additionally, mAP metrics indicate an increase as well (i.e. $0.5 \mathrm{~m}, 1 \mathrm{~m}, 2 \mathrm{~m}$, and $4 \mathrm{~m}$ ) and we see a significant improvement in the NDS.

Moreover, we show the effectiveness of our rangeguided backbone to reduce the receptive field for larger distances and for smaller objects. For range $[30 \mathrm{~m}, 50 \mathrm{~m}]$ over all classes we achieve NDS of 0.485 when the guidance mechanism is applied, and 0.478 when omitted while using the heavily optimized backbone of CBGS [32]. Additionally, we observe an increase in mAP in four out of the five smallest classes (i.e barrier, traffic cone, cyclist, and motorcycle) for the entire range, resulting in an increase from $0.574 \mathrm{mAP}$ to $0.576 \mathrm{mAP}$ over all classes.

\section{Conclusions}

In this paper, we explore the potential of the Cylindrical coordinates system for representing LiDAR point clouds in outdoor scenes. We present a novel end-to-end framework for 3D object detection in those coordinates together with a novel range-guided backbone that can be readily added to other pipelines. Our method achieves powerful results on the highly challenging nuScenes dataset and lays the cornerstone for further Cylindrical methods in this domain. 


\section{References}

[1] J. Behley, M. Garbade, A. Milioto, J. Quenzel, S. Behnke, C. Stachniss, and J. Gall. SemanticKITTI: A Dataset for Semantic Scene Understanding of LiDAR Sequences. In Proc. of the IEEE/CVF International Conf. on Computer Vision (ICCV), 2019. 2

[2] Alex Bewley, Pei Sun, Thomas Mensink, Dragomir Anguelov, and Cristian Sminchisescu. Range conditioned dilated convolutions for scale invariant $3 \mathrm{~d}$ object detection, 2020. 2

[3] Holger Caesar, Varun Bankiti, Alex H. Lang, Sourabh Vora, Venice Erin Liong, Qiang Xu, Anush Krishnan, Yu Pan, Giancarlo Baldan, and Oscar Beijbom. nuscenes: A multimodal dataset for autonomous driving. arXiv preprint arXiv:1903.11027, 2019. 7

[4] R. Q. Charles, H. Su, M. Kaichun, and L. J. Guibas. Pointnet: Deep learning on point sets for 3d classification and segmentation. In 2017 IEEE Conference on Computer Vision and Pattern Recognition (CVPR), pages 77-85, 2017. 1

[5] L. Chen, G. Papandreou, I. Kokkinos, K. Murphy, and A. L. Yuille. Deeplab: Semantic image segmentation with deep convolutional nets, atrous convolution, and fully connected crfs. IEEE Transactions on Pattern Analysis and Machine Intelligence, 40(4):834-848, 2018. 2

[6] Qi Chen, Lin Sun, Zhixin Wang, Kui Jia, and Alan Yuille. Object as hotspots: An anchor-free 3d object detection approach via firing of hotspots, 2020. 7

[7] Xiaozhi Chen, Huimin Ma, Ji Wan, Bo Li, and Tian Xia. Multi-view 3d object detection network for autonomous driving. In Proceedings of the IEEE Conference on Computer Vision and Pattern Recognition (CVPR), July 2017. 1, 2

[8] Yilun Chen, Shu Liu, Xiaoyong Shen, and Jiaya Jia. Fast point r-cnn. In Proceedings of the IEEE/CVF International Conference on Computer Vision (ICCV), October 2019. 1

[9] Mark Everingham, Luc Gool, Christopher K. Williams, John Winn, and Andrew Zisserman. The pascal visual object classes (voc) challenge. Int. J. Comput. Vision, 88(2):303-338, June 2010. 7

[10] Andreas Geiger, Philip Lenz, and Raquel Urtasun. Are we ready for autonomous driving? the kitti vision benchmark suite. In Conference on Computer Vision and Pattern Recognition (CVPR), 2012. 3

[11] Sylvain Gugger. The 1cycle policy. https://sgugger.github.io/the-1cycle-policy.html. 8

[12] Kaiming He, X. Zhang, Shaoqing Ren, and Jian Sun. Deep residual learning for image recognition. 2016 IEEE Conference on Computer Vision and Pattern Recognition (CVPR), pages 770-778, 2016. 4

[13] Jason Ku, Melissa Mozifian, Jungwook Lee, Ali Harakeh, and Lake Steven Waslander. Joint 3d proposal generation and object detection from view aggregation. Intelligent Robots and Systems (IROS), 2018. 1, 2

[14] Alex H. Lang, Sourabh Vora, Holger Caesar, Lubing Zhou, Jiong Yang, and Oscar Beijbom. Pointpillars: Fast encoders for object detection from point clouds. In CVPR, 2019. 1
[15] Hei Law and Jia Deng. Cornernet: Detecting objects as paired keypoints. In Proceedings of the European Conference on Computer Vision (ECCV), September 2018. 6

[16] Zhidong Liang, Ming Zhang, Zehan Zhang, Xian Zhao, and Shiliang Pu. Rangercnn: Towards fast and accurate $3 \mathrm{~d}$ object detection with range image representation, 2020. 1, 2

[17] T. Lin, P. Goyal, R. Girshick, K. He, and P. Dollár. Focal loss for dense object detection. In 2017 IEEE International Conference on Computer Vision (ICCV), pages 2999-3007, 2017. 7

[18] Ilya Loshchilov and Frank Hutter. Decoupled weight decay regularization. In 7th International Conference on Learning Representations, ICLR 2019, 2019. 8

[19] Gregory P. Meyer, Ankit Laddha, Eric Kee, Carlos VallespiGonzalez, and Carl K. Wellington. Lasernet: An efficient probabilistic $3 \mathrm{~d}$ object detector for autonomous driving. In The IEEE Conference on Computer Vision and Pattern Recognition (CVPR), 2019. 2

[20] Adam Paszke, Sam Gross, Francisco Massa, Adam Lerer, James Bradbury, Gregory Chanan, Trevor Killeen, Zeming Lin, Natalia Gimelshein, Luca Antiga, Alban Desmaison, Andreas Kopf, Edward Yang, Zachary DeVito, Martin Raison, Alykhan Tejani, Sasank Chilamkurthy, Benoit Steiner, Lu Fang, Junjie Bai, and Soumith Chintala. Pytorch: An imperative style, high-performance deep learning library. In $\mathrm{H}$. Wallach, H. Larochelle, A. Beygelzimer, F. d'Alché-Buc, E. Fox, and R. Garnett, editors, Advances in Neural Information Processing Systems 32, pages 8024-8035. Curran Associates, Inc., 2019. 7

[21] Charles Ruizhongtai Qi, Li Yi, Hao Su, and Leonidas J Guibas. Pointnet++: Deep hierarchical feature learning on point sets in a metric space. In I. Guyon, U. V. Luxburg, S. Bengio, H. Wallach, R. Fergus, S. Vishwanathan, and R. Garnett, editors, Advances in Neural Information Processing Systems, volume 30, pages 5099-5108. Curran Associates, Inc., 2017. 1

[22] Ernest Cheung Qi Chen, Lin Sun and Alan L. Yuille. Every view counts: Cross-view consistency in $3 \mathrm{~d}$ object detection with hybrid-cylindrical-spherical voxelization. In $\mathrm{Ad}$ vances in Neural Information Processing Systems 33 preproceedings (NeurIPS), 2020. 2, 7, 8

[23] Shaoshuai Shi, Chaoxu Guo, Li Jiang, Zhe Wang, Jianping Shi, Xiaogang Wang, and Hongsheng Li. Pv-renn: Point-voxel feature set abstraction for $3 \mathrm{~d}$ object detection. In IEEE/CVF Conference on Computer Vision and Pattern Recognition (CVPR), June 2020. 1, 2

[24] Shaoshuai Shi, Zhe Wang, Jianping Shi, Xiaogang Wang, and Hongsheng Li. From points to parts: 3 d object detection from point cloud with part-aware and part-aggregation network. IEEE Transactions on Pattern Analysis and Machine Intelligence, PP:1-1, 02 2020. 1, 2

[25] P. Wang, P. Chen, Y. Yuan, D. Liu, Z. Huang, X. Hou, and G. Cottrell. Understanding convolution for semantic segmentation. In 2018 IEEE Winter Conference on Applications of Computer Vision (WACV), pages 1451-1460, 2018. 2

[26] Yan Yan, Yuxing Mao, and Bo Li. Second: Sparsely embedded convolutional detection. Sensors, 18(10):3337, Oct 2018. 1, 2, 5 
[27] Bin Yang, Wenjie Luo, and Raquel Urtasun. Pixor: Realtime $3 \mathrm{~d}$ object detection from point clouds. In Proceedings of the IEEE Conference on Computer Vision and Pattern Recognition (CVPR), June 2018. 1

[28] Tianwei Yin, Xingyi Zhou, and Philipp Krähenbühl. Centerbased 3d object detection and tracking, 2020. 2, 5, 6, 7, 8

[29] Wenwei Zhang, Kai Chen, Zhe Wang, Jianping Shi, and Chen Change Loy. Mmdetection3d. https://github.com/open-mmlab/mmdetection3d. 7

[30] Yang Zhang, Zixiang Zhou, Philip David, Xiangyu Yue, Zerong Xi, Boqing Gong, and Hassan Foroosh. Polarnet: An improved grid representation for online lidar point clouds semantic segmentation. In Proceedings of the IEEE/CVF Conference on Computer Vision and Pattern Recognition (CVPR), June 2020. 2

[31] Y. Zhou and O. Tuzel. Voxelnet: End-to-end learning for point cloud based $3 \mathrm{~d}$ object detection. In 2018 IEEE/CVF Conference on Computer Vision and Pattern Recognition, pages 4490-4499, 2018. 1, 2, 5

[32] Benjin Zhu, Zhengkai Jiang, Xiangxin Zhou, Zeming Li, and Gang Yu. Class-balanced grouping and sampling for point cloud 3d object detection, 2019. 2, 5, 7, 8 